\title{
Human Verification \\ Using 3D-gray-scale Face Image
}

\author{
Ryutaro ITO Kazuo NAKAZAWA Masato NAKAJIMA
}

Faculty of Science and Technology, Keio University

Hiyosi 3-14-1, Kouhoku ward Yokohama city, Japan

\begin{abstract}
In recent years, many different techniques have been proposed to verify human face. Most of them used gray-scale image and some of them used range image. Because the two types of image include different infornation, their combination can increase the correct verification rate. The paper propose a human face verification method using a 3D-gray-scale imaging technique. First, we developed an effectiveness procedure for obtaining a 3D-gray-scale face image based on a 2D-gray-scale image and a range image. Then we propose a human face verification technique using the subspace method: to verify whether the observed person is himself, distance is calculated between the face data of the person and the subspace of the registered data. In order to verify the effectiveness of our method, we applyed this method to 18 people. The result of experiment shows effectiveness of the method.
\end{abstract}

\section{Introduction}

In recent years, because automatic verification system is required as a security system, so many different identification and verification techniques have been proposed using human features, for example the face or the fingerprint. Especially the human verification using face information is a common way people recognize other people, so it is considered that the personal verification using human face is consistent with good reason. Actually, many different methods of human face identification and verification methods have been proposed. Most of them are only using 2D-gray-scale images $[1,2,3,4,5,6]$, and some of them are only using range images. We already reported an identification method using range information[7]. However, because the two types of images include different information, it is expected that using both information increases the correct verification rate[8].

In this paper, we propose a verification method using 3D-gray-scale images obtained from 2D-gray-scale and range information. Employing the 3D-gray-scale images, the calibration of the direction of the face can be easily performed and the normalization of the face size can be omitted. When only the 2D-gray-scale images are used, the calibration of the face direction and the normalization of the face size is difficult. Accordingly most of the methods proposed so far presupposed face images in which the human face direction is just the front and human face size is fixed.

In this paper, first, we propose an efficient procedure for obtaining the $3 \mathrm{D}$-gray-scal images. Then we propose a verification method using these images. For acquiring $2 \mathrm{D}-$ gray-scale image, we use infrared lighting to avoid the influence of another lighting. Then the system can be used in a room under a fluorescent light. In this method, the face data are acquired from the front. But the direction of the face varies, because of loose control of data acquisition, so the calibration of the face direction is indispensable. We use the calibration method we developed before[7].

The purpose of the verification method is to verify whether the observed person is the registered person himself. The verification system verifies whether the person's face data corresponds to the registered data retrieved by the ID number input by the person. Such situation could happen at the entrance of a secret room and so on. 
To verify human face, we use the subspace method[2, 9]. Many identification methods are known among which eigen space method[9] and subspace method[2] show good results. We chose subspace method because the training for a person to be registered can be done only using that person's data. The verification is done by calculating the distance from the observed face data to the registered personal facial subspace. If the distance is shorter than the threshold, the face data is judged belonging to that subspace.

We applied this method to 18 people, and the result of the experiment shows the effectiveness of the method.

\section{Acquisition of the 3D-gray-scale Face Image}

The sensor system to acquire the $3 \mathrm{D}$-gray-scale images is shown in Fig.1. The system consists of a FG(Fiber Grating) Vision Sensor[10], and an infrared lighting equipment. The FG sensor is composed of a laser diode, Fiber Gratings, and a CCD-camera. The system can acquire a range image and a $2 \mathrm{D}$-gray-scale image at almost the same time, by switching on the laser and the infrared lighting alternately. FG is made from optical fibers aligned side by side. In Fig.1, the two sheets of FG are piled up so that the grating direction of one shect of $F G$ is vertical and that of the other is horizontal. When the laser ray is irradiated into the FG in Fig.1 vertically, the array of optical spots is projected on the reference screen which is located behind the human face. In this optical arrangement in Fig.1, when a spot is projected on a human face, the spot reflected and imaged on the image plane moves horizontally by a specific amount $\delta$ from the image point $(x, y)$ of the spot on the reference screen. In this arrangement, the positions of the spot $(X, Y, Z)$ on the face are calculated by the following equations.

$$
\begin{aligned}
Z & =\frac{h^{2} \delta}{d f+h \delta} \\
X & =\frac{x+\delta}{f}(h-Z) \\
Y & =\frac{y}{f}(h-Z),
\end{aligned}
$$

where $h$ is the distance between the lens and the screen, and $d$ is the distance between the FG and the lens, and $f$ is the distance between the lens and the image plane. The spot-projected image and the 2D-gray-scale image acquired by this sensor are shown in Fig.2. Fig.3 shows the 3D-gray-scale image obtained by mapping the 2D-gray-scale image onto the range image.

\section{Calibration of the Face Direction}

Although the face data are taken from the front, the true direction of the face varies within certain extent. Therefore, it is important to calibrate the direction of the face of the 3D-gray-scale image. Calibration of the face direction is done as the following, using the method we developed before[7] and illustrated in Fig.4.

1. The nose tip is searched for from the range information.

2. The normal vectors are calculated from the range information.

3. These normal vectors of the face surface within $5 \mathrm{~cm}$ from the nose tip are projected to a Gaussian Sphere and the Gaussian Sphere is dissected into three regions as Fig. 4 shows. 
4. The face data are rotated around the $x$ axis and $y$ axis so as the centroid of the normal vectors included in regionl coincides with the center of the Gaussian Sphere.

5. The face data are rotated around the $z$ axis, so as the left centroid $G l$ and the right centroid $G r$ included in region 2 becomes symmetric with respect to y axis.

6. The process from 1 to 5 is repeated three times sufficiently to calibrate the direction of the face.

7. The position of the face is calibrated by placing the nose tip at the origin of the coordinate axis.

switching circuit

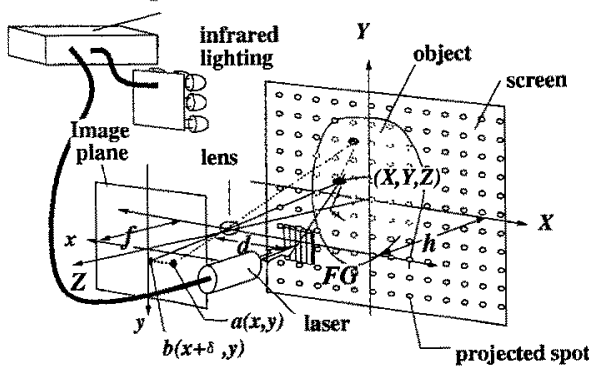

Fig.1. The optical arrangement of the sensor.

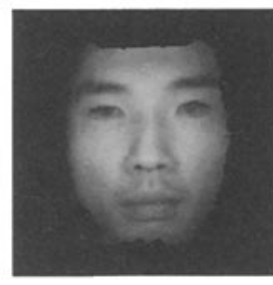

(a) Front face

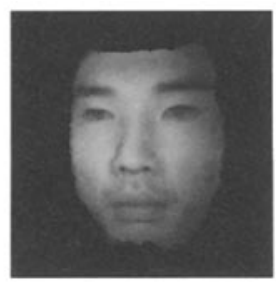

(b) Rotated face

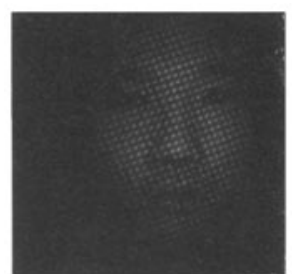

(a) Spot-projected image

Fig.2. Acquired images.

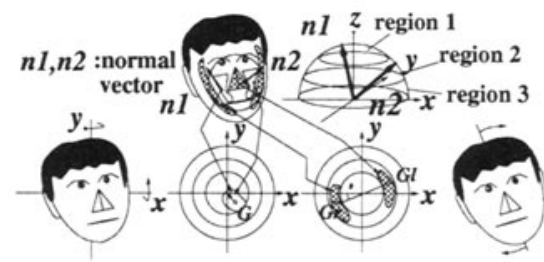

calibration around $\mathrm{x}$ axis and $\mathrm{y}$ axis

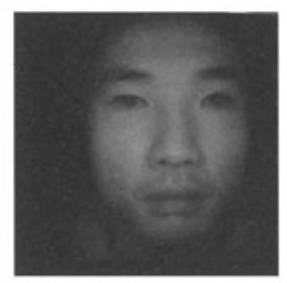

(b) 2D-gray-scale image
Fig.4. Calibration of the face direction.

\section{Verification Using Subspace}

Verification employs the subspace method. In this method, the verification procedure is carried out only by calculating the distance in the feature vector space between the observed face data and the subspace of the registered data retrieved by the ID number input by the person to be verified.

If the distance from the observed data $x$ to the subspace of the registered data is less than the threshold, the observed person is judged to be the person with the ID number. The concept of the verification method using subspace is shown in Fig.5. In Fig.5, $V_{i}$ is the subspace of the face data category $\xi_{i}$ where $i$ is the ID number of the registered person. If the projection matrix to the subspace $V_{i}$ is defined as $P_{i}$, the distance between the $x$ and subspace $V_{i}$ is defined by the following equation.

$$
\begin{aligned}
\operatorname{Dist}\left(V_{i}, x\right) & =\left\|x-\left(P_{i}\left(x-\mu_{i}\right)+\mu_{i}\right)\right\| \\
& =\left\|\left(I-P_{i}\right)\left(x-\mu_{i}\right)\right\| .
\end{aligned}
$$


In this cquation $\mu_{i}$ is the average vector of category $\xi_{i}$. Projection matrix $P_{i}$ is defined by the following

$$
P_{i}=V_{i} V_{i}^{T}
$$

where matrix $V_{i}$ denotes the set of basis vectors for subspace $V_{i}$.

\section{Process of Verification}

The flowchart of verification process is shown in Fig.6. As shown in the Fig.6, the process is divided into two phases; training and verification. Training is carried out when the person is registered. Verification is performed when the person is verified.

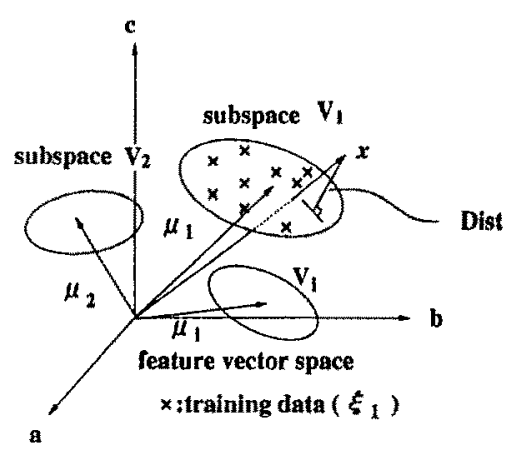

Fig.5. Verification using subspace.

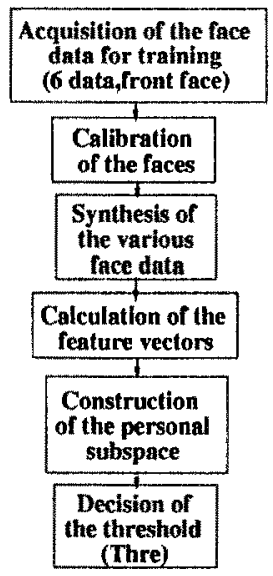

(a)Training

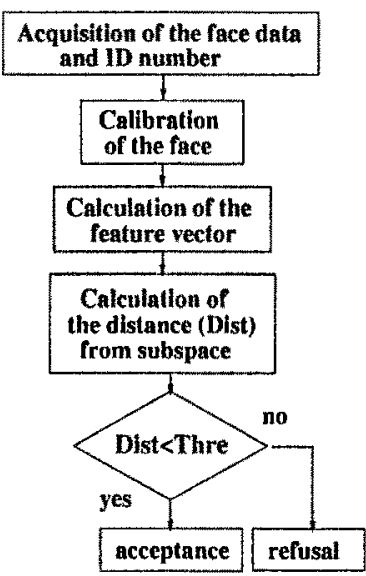

(b) Verification

Fig.6. Flowchart of the process of verification.

\subsection{Synthesis of the Various Face Data for Training}

Because of the slip which occurs when the data is calibrated and the variation of the brightness caused by varied direction of the face, the face data to be verified risks to be largely different from the data acquired in training. In order to avoid verification error caused by such discrepancies, a set of data, representing expected variation of the component parameters of the feature vectors, are synthesized from the front face data obtained in registration. These data are synthesized, considering slips of eight direction (up-and-down slip $\pm 3 \mathrm{~mm}$, and right and left slip $\pm 3 \mathrm{~mm}$ ), and the difference of distribution of brightness caused by the rotation of eight direction (up-and-down rotation $\pm 10^{\circ}$ and left and right rotation $\pm 8^{\circ}$ ).

\subsection{Calculation of the Feature Vector}

Specification of the feature vector components is shown in Fig. 7 and an example of the set of the feature vector components used in verification is depicted in Fig.8. The feature vector components are defined as follows. The normal vectors within the region are calculated from the $3 \mathrm{D}$-gray-image for the mosaicked surface segments. In the same way the gray-scale information, after normalization, is calculated for the finer mosaicked surface segments. The normalization is done by the following equation.

$$
\bar{f}_{x y}=\left(f_{x y}-\mu\right) / \sigma,
$$


where, $f_{x y}$ is the brightness of the pixel and $\bar{f}_{x y}$ is the normalized brightness. $\mu$ is the average brightness within the region and $\sigma$ is the standard deviation within the region. The number of normal vector mosaic is 98 and each vector contains two parameter $n_{x}, n_{y}$, so the number of dimension of normal vectors becomes 196 . The number of gray-scale mosaic is 202. Accordingly the number of dimension of the feature vector becomes 398 .

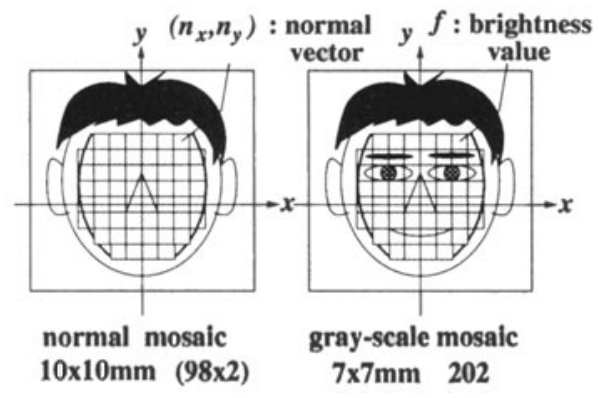

Fig.7. Specification of the feature vector components.

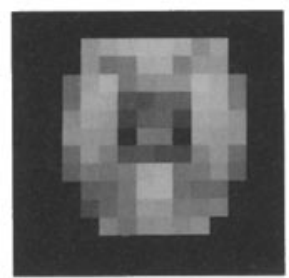

(a) Normal vector mosaic image $10 \times 10 \mathrm{~mm}$

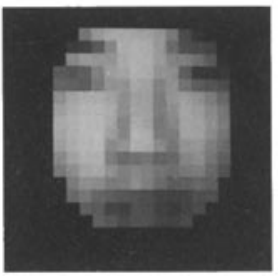

(b) Gray-scale mosaic image $7 \times 7 \mathrm{~mm}$ Fig.8. Example of the feature vector.

\subsection{Construction of the Personal Subspace}

Let $x_{i k}$ denote the $k$ th feature vector of the category $\xi_{i}$ and $N$ denote the dimension of the feature vector. The process of construction of the subspace is as the following.

1. Calculate the mean $\mu_{i}$ and covariance matrix $R_{i}$ of the category.

$$
\begin{aligned}
& \mu_{i}=\frac{1}{M} \sum_{k=1}^{M} x_{i k} \\
& R_{i}=\frac{1}{M} \sum_{k=1}^{M}\left(x_{i k}-\mu_{i}\right)\left(x_{i k}-\mu_{i}\right)^{T} .
\end{aligned}
$$

2. Calculate eigenvalues by finding such an orthogonal matrix

$$
U_{i}=\left\{v_{i 1}, \cdots, v_{i m}, \cdots, v_{i N}\right\}
$$

as

$$
R_{i}=U_{i} \Sigma_{i} U_{i}^{T}
$$

is satisfied with the diagonal matrix

$$
\Sigma_{i}=\operatorname{diag}\left(\lambda_{i 1}, \lambda_{i 2}, \cdots, \lambda_{i m}, \cdots, \lambda_{i N}\right) .
$$

$v_{i m}$ is the eigenvector belonging to the eigenvalue $\lambda_{i m}$, for which

$$
\lambda_{i 1} \geq \lambda_{i 2} \geq \cdots \geq \lambda_{i m} \cdots \geq \lambda_{i N} \geq 0 .
$$

As the bases of the subspace $V_{i}$ we chose

$$
V_{i}=\left\{v_{i 1}, v_{i 2} \cdots, v_{i n}\right\},
$$

where

$$
\sum_{m=1}^{n} \lambda_{i m}=0.99 \cdot \sum_{m=1}^{N} \lambda_{i m} \text {. }
$$

3. At the end of this process, the threshold is determined for the distance(defined in (4)) below which the verification declares acceptance. Using the data used to determine the subspace, we calculate the distance from each of the feature vectors to the subspace. Then the standard deviation $\sigma$ of the distance is calculated, and $3 \sigma$ was decided to be the threshold $T_{i}$. 


\subsection{Verification}

The process of verification proceeds as follows.

1. Projection matrix $P_{i}$ is calculated by equation (6) for the subspace $V_{i}$ specified by the ID number input by the person to be verified.

2. The distance $\operatorname{Dist}\left(V_{i}, x\right)$ from the observed feature vector $x$ to the subspace $V_{i}$ is calculated by equation (5).

3. If and only if the following condition is satisfied, the measured face data $x$ is judged belonging to the subspace $V_{i}$. Then the person is accepted as the declared person.

$$
\operatorname{Dist}\left(V_{i}, x\right) \leq T_{i}
$$

\section{Experiment}

In order to verify the effectivencss of our proposal, we applied this method to 18 people. The face data were acquired from the front of the face. The subject had to stand with their feet on the footprints painted on the floor, and to manage to place his nose reflected in a mirror in the $5 \mathrm{~cm} \times 5 \mathrm{~cm}$ square area marked on the mirror located at the image plane position. The number of the face data for registration was 6 data per person and the training data were synthesized from these, and the number of training data became $486(6$ data $\times 9 \times 9)$. The number of the face data used for verification is 8 data per person, so that the number of test data became $144(18$ person $\times 8$ data). Some examples of 3D-gray-scale images used for the experiment are shown in Fig.9.
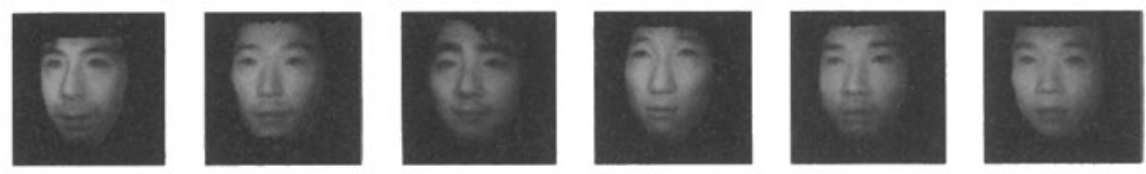

Fig.9. The examples of 3D-gray-scale images.

\section{Result of Verification Experiment}

We summarize the result of verification experiment in Table1. The dimension of the subspace depends on the person, but the average dimension was 22. The Table1 indicates 2 cases. The left column was the case in which the person input his own ID number. In this case, the acceptance rate implies the fraction of the results where the system correctly judged the person to be the person designated by the input ID. The right column was the case in which the person input some other person's ID number. In this case, the refusal rate implies the fraction of the results where the system correctly judged the person not to be the person designated by the input ID.

The Fig.10 and Fig.11 are examples of the 2D-gray-scale images used for obtaining 3D-gray-scale images. All of the data in Fig. 10 resulted in correct acceptance. And all of the data in Fig. 11 resulted in incorrect refusal. Although situations like those in Fig.11, hardly happen with the subject control imposed in the experiment as stated in section 6 . The Fig.12 shows the relation between the correct acceptance rate and rotation angle of the face.

Finally Fig.13 shows the relation between correct verification rate and threshold. In the experiment so far mentioned, the threshold of verification was fixed at $3 \sigma$. 
However the threshold seems better chosen between $3.0 \sigma$ and $3.5 \sigma$ for higher correct acceptance rate without lowering correct refusal rate.

Table 1.Correct Verification Rate

\begin{tabular}{|c|c|}
\hline Acceptance Rate & Refusal Rate \\
\hline $139 / 144(96.5 \%)$ & $2448 / 2448(100 \%)$ \\
\hline
\end{tabular}

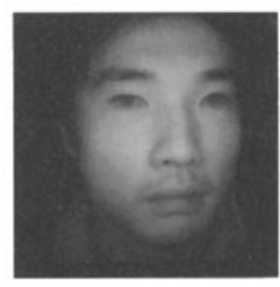

(a) Left $8.0^{\circ}$

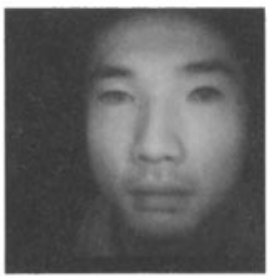

(b) Right $8.0^{\circ}$

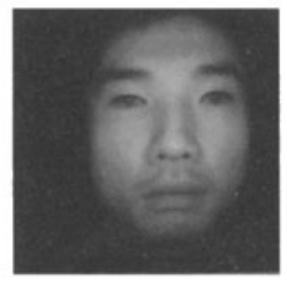

(c) Up $7.0^{\circ}$

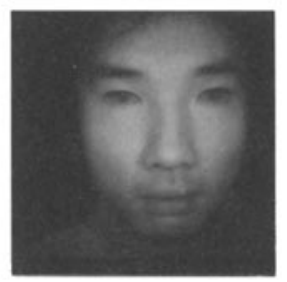

(d) Down $7.0^{\circ}$

Fig.10. The examples of face images which are verified correctly.

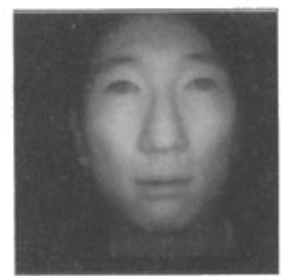

(a) Up $8.5^{\circ}$

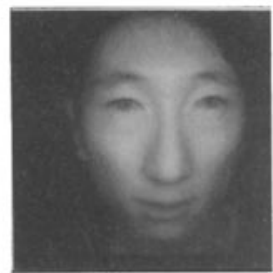

(b) Down $9.2^{\circ}$

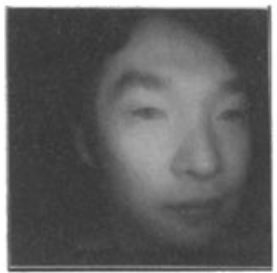

(c) Left $13.1^{\circ}$

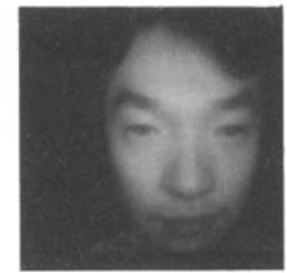

(d) Down $10.4^{\circ}$

Fig.11. The examples of face images which are verified incorrectly.
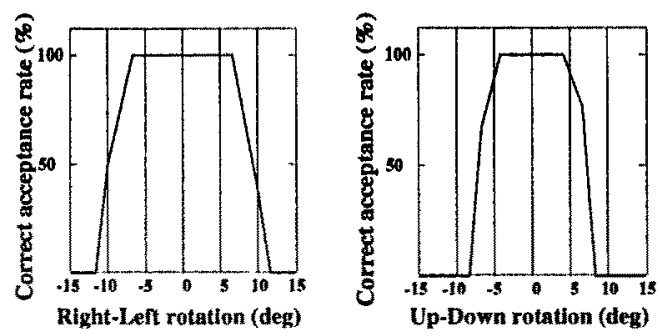

Fig.12. Relation between correct acceptance rate and rotation angle of the face.

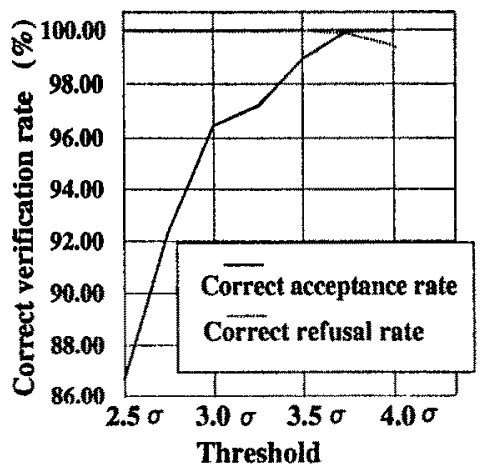

Fig.13. The relation between the correct verification rate and threshold. 


\section{Conclusion}

We proposed a human face verification method using the 3D-gray-scale imaging technique. To obtain the 3D-gray-scale image, we developed a new sensor which can obtain spot-projected image and 2D-gray-scale image at almost the same time. By fusing the latter with the range image calculated from the former, the $3 \mathrm{D}$-gray-scale image is efficiently obtained. Using this $3 \mathrm{D}$-gray-scale image, we proposed a new verification mothod. The proposed method first calibrates the face dircetion by using the range image accorcling to the the method developed in [7]. The variation of the distribution of the brightness caused by the rotation of the face, however, still exists. We employed the subspace method so that the verification could be done correctly in spite of such variation. We acquired in advance the feature vector data from the front of the face of the persons to be registered and synthesized the data representing expected variation in the component parameters of the feature vector. In verification the subspaces are constructed from these data and the distance is calculated between the face data of the person to be verified and the subspace of the registered data retrieved by the ID number input by the observed person. And the person is verified as the person designated by the ID number if and only if the distance is less than a threshold.

We made an experiment to verify the effectiveness of this method. We applied this method to 18 people and obtained more than $98 \%$ of correct verification rate and almost $100 \%$ of correct refusal rate.

\section{References}

[1] Makoto Kosugi: "Human-Face Recognition Using Mosaic Pattern and Noural Notworks", IEICE Trans(D-II),J-76-D-II,No.6,pp.1132-1139(1993)

[2] Yoshie Komatsu: "Orientation Invariant Face Extraction and Recognition based on Subspace Method", TECHNICAL REPORT OF IEICE,PRU95-191,(1996-01)

[3] Roberto Brunelli, Tomaso Poggio: "Face Recognition: Features versus Templates", IEEE,Trans,Patt.Anal.Machine Intell.,Vol.12,No.10,pp.1042-1052,1993

[4] Yong-Qing Cheng,Ke Liu,Jing-Yu Yang: "A NOVEL FrATURE EXTRACTION METHOD FOR IMAGE RECOGNITION BASED ON SIMILAR DISCRIMINANT FUNXTION(SDF)", Pattern Recognition, Vol.26,No.1,pp.115-125,1993

[5] David J. Beymer: "Face Recognition Under Varying Pose", In Proceedings IEEE Conf. on Computer Vision and Pattern Recognition, pp.756-761,1994

[6] David Beymer, Tomaso Poggio: "Face Recognition From One Example View", In Proceedings IEEE Conf, on Computer Vision and Pattern Recognition, pp.500-507,1995

[7] Kenji Terada,Jun'ichi Yamaguchi,Masato Nakajima : "An Automatic Identification of Human Faces using Fiber Grating Vision Sensor", T.IEE Japan,Vol.113C,No.12,pp.1072-1078,(1993)

[8] Shinya Imabeppu: "Individual Identification by Unifying Profile and Front-View Images", TECHNICAL REPORT OF IEICE,PRU94-148,(1995-03)

[9] Hiroshi Murase: "3D Object Recognition from Appearance-Parametric Eigen space Method-", T.IEE Japan, Vol.J77-D-II,No.11,pp.2179-2187(1994)

[10] Kazuo Nakazawa: "Development of Compact Vision Sensor by Fusion of 2-D Intensity Image and Sparse Range Image", T.IEE Japan,Vol.115 ,No.3,pp.389-395(1995) 